\title{
Helicobacter Pylori, Non-Ulcer Dyspepsia, and Childhood Recurrent Abdominal Pain
} A review of: Moayyedi P, Soo S, Deeks J, Forman D, Mason J, Innes M, Delaney B on behalf of the Dyspepsia Review Group
2000 Systematic review and economic evaluation of Helicobacter pylori eradication treatment for non-ulcer dyspepsia. BMJ 321:659-664

$\mathrm{I}_{\mathrm{d}}^{\mathrm{n}}$ adults, there is compelling evidence that Helicobacter pylori infection causes peptic ulcer disease (1). The relationship between $H$. pylori infection and non-ulcer dyspepsia, however, is more controversial. Non-ulcer dyspepsia is defined as persistent or recurrent upper abdominal discomfort, of at least 12 weeks duration, with no evidence of organic disease on investigation. The prevalence of non-ulcer dyspepsia in adults is around $10 \%$ and $H$. pylori infection is slightly more prevalent in adults with non-ulcer dyspepsia compared with healthy controls (2).

Two systematic reviews have provided conflicting results regarding the effect of $H$. pylori eradication on symptom resolution in patients with non-ulcer dyspepsia $(2,3)$. These reviews, however, have been criticized on methodological grounds. A more recent meta-analysis by Moayyedi et al (4) has addressed the limitations of the previous reviews. The authors used a comprehensive search strategy to identify all available randomized, placebo controlled treatment trials. Strict eligibility criteria were applied; that is, trials were included if dyspepsia was defined based on published criteria, patients with peptic ulcer or esophagitis were excluded, the intervention $(H$. pylori eradication treatment) was effective and validated measures were used to assess clinical and economic outcomes. The primary outcome measure was symptom relief at 12 months. The authors tested for heterogeneity of results across studies and also calculated the number-needed-to-treat (NNT): the number of patients with non-ulcer dyspepsia that would need to be treated with $H$. pylori eradication

\section{Colin Macarthur}

therapy in order to cure one patient. Last, the cost-effectiveness of eradicating $H$. pylori infection in patients with non-ulcer dyspepsia was assessed. For this analysis, eradication treatments (antibiotics + proton pump inhibitor) were compared with antacid treatment.

The authors pooled the results of nine placebo controlled treatment trials (2,541 patients) and showed a small, but significant, benefit of $H$. pylori eradication. On average, at 12 months, $36 \%$ were improved in the treatment group compared with $28 \%$ improvement in the placebo group (relative risk reduction $9 \%$; 95\% confidence interval $4 \%$ to $14 \%$ ). The NNT to cure one patient with non-ulcer dyspepsia was 15 patients $(95 \%$ confidence interval 10 to 31 patients) and the economic analysis demonstrated a cost of 56 pounds sterling (\$84 US) for each month free from dyspepsia.

There are some similarities between non-ulcer dyspepsia in adults and recurrent abdominal pain in children. For example, the prevalence of recurrent abdominal pain is around $10 \%$ in unselected school children. Furthermore, the relationship between $H$. pylori infection and childhood recurrent abdominal pain is also controversial. The evidence for an association, if any, between $H$. pylori infection and recurrent abdominal pain is limited to a few observational epidemiologic studies. A recent review of five case-control studies showed inconsistent results, with one study suggesting that $H$. pylori infection was significantly more common in children with recurrent abdominal pain (compared with healthy controls), whereas another study suggested that $H$. pylori infection was significantly less common in recurrent abdominal pain (5). This review, which was limited to a single database, did not identify any randomized, eradication trials in children.

Therefore, based on the available evidence, it would not seem appropriate to recommend $H$. pylori eradication therapy for children with recurrent abdominal pain. The Moayyedi et al study, however, highlights the need for a systematic and comprehensive review of the literature on the association between $H$. pylori infection and childhood recurrent abdominal pain.

The best evidence for (or against) an association between $H$. pylori infection and recurrent abdominal pain will come from comparison studies, for example, observational studies with healthy control groups or controlled treatment trials (preferably randomized, double blind, placebo controlled studies). Such data will help clarify the relationship between $H$. pylori infection and childhood recurrent abdominal pain.

\footnotetext{
1. Veldhuyzen van Zanten S 2000 Treating non-ulcer dyspepsia and H pylori. BMJ 321:648-649

2. Jaakkimainen L, Boyle E, Tudiver F 1999 Is Helicobacter pylori associated with non-ulcer dyspepsia and will eradication improve symptoms? A metaanalysis. BMJ 319:1040-1044

3. Danesh J, Pounder E 2000 Eradication of Helicobacter pylori and non-ulcer dyspepsia. Lancet 355:766-767

4. Moayyedi P, Soo S, Deeks J, Forman D, Mason J, Innes $\mathrm{M}$, Delaney $\mathrm{B}$ on behalf of the Dyspepsia Review Group 2000 Systematic review and economic evaluation of Helicobacter pylori eradication treatment for non-ulcer dyspepsia. BMJ 321:659-664

5. Macarthur C 1999 Helicobacter pylori infection and childhood recurrent abdominal pain: lack of evidence for a cause-and-effect relationship. Can J Gastroenterol 13:607-610
}

The Hospital for Sick Children

Paediatric Outcomes Research Team

Department of Paediatrics

555 University Avenue

Toronto, Ontario M5G $1 X 8$, Canada 